Pesq. Vet. Bras. 37(5):453-458, maio 2017 DOI: $10.1590 / \mathrm{S} 0100-736 \mathrm{X} 2017000500005$

\title{
Termografia infravermelha da superfície ocular como indicador de estresse em suínos na fase de creche ${ }^{1}$
}

\author{
Lina Fernanda Pulido-Rodríguez ${ }^{2}$, Evaldo Antonio Lencioni Titto ${ }^{2}$, Fábio Luís \\ Henrique $^{2}$, Ana Luisa Silva Longo ${ }^{2}$, Henrique Barbosa Hooper ${ }^{2}$, Thuanny Lúcia \\ Pereira $^{2}$, Alfredo Manuel Franco Pereira ${ }^{3}$ e Cristiane Gonçalves Titto ${ }^{2 *}$
}

\begin{abstract}
Pulido-Rodríguez L.F., Titto E.A.L., Henrique F.L., Longo A.L.S., Hooper H.B., Pereira T.L., Pereira A.M.F. \& Titto C.G. 2017. [Infrared thermography of the ocular surface as stress indicator for piglets postweaning.] Termografia infravermelha da superfície ocular como indicador de estresse em suínos na fase de creche. Pesquisa Veterinária Brasileira 37(5):453-458. Laboratório de Biometeorologia e Etologia, Faculdade de Zootecnia e Engenharia de Alimentos, Universidade de São Paulo, Av. Duque de Caxias Norte 225, Campus Fernando Costa, Pirassununga, SP 13635-900, Brazil. E-mail: crisgtitto@usp.br

Weaning is a critical phase in pigs' life due to maternal separation and the introduction of a new diet. Infrared thermography measurement taken in the ocular region appears to be a reliable indicator of the stress condition of the pig. The aim of this study was to evaluate the relationship between ocular surface temperature by infrared thermography and cortisol in piglets post weaning. Sixty-six piglets were evaluated once a week, during 7 weeks, in two periods of the day (7am and $15 \mathrm{pm})$ and ocular surface temperature and dorsal surface temperature were collected using a thermographic camera and a laser surface thermometer, respectively. Saliva was also collected to determine salivary cortisol. Statistical analysis included fixed effects of week and period of the day and their interaction, and relationship between thermography, dorsal surface temperature and cortisol were done by Pearson's correlations with $5 \%$ significance level. Salivary cortisol did not differ between periods, but it was higher in the first three weeks after weaning $(\mathrm{P}<0.05)$. During the first two weeks after weaning cortisol presented high and positive correlation $(\mathrm{P}<0.05)$ between ocular surface temperature $(0.89)$ and dorsal surface temperature $(0.80)$. The two superficial temperatures had a moderate and positive association $(\mathrm{r}=0.41 ; \mathrm{P}<0.0001)$ during all experiment. This study highlights that the ocular surface temperature obtained through infrared thermography can be a superficial body temperature indicators, besides being a non-invasive and fast method of measurement. However, more research is needed to deepen the relationship between ocular surface temperature and cortisol during chronic stress.

INDEX TERMS: Infrared thermography, ocular surface, stress indicator, piglets postweaning, animal welfare, body temperature, infrared image, salivary cortisol, weaning.
\end{abstract}

RESUMO.- Desmama é uma fase crítica na vida do suíno devido a separação materna e a introdução de uma dieta seca. A termografia infravermelha medida na região ocu-

\footnotetext{
${ }^{1}$ Recebido em 10 de janeiro de 2016.

Aceito para publicação em 4 de setembro de 2016.

${ }^{2}$ Laboratório de Biometeorologia e Etologia, Faculdade de Zootecnia e Engenharia de Alimentos, Universidade de São Paulo (USP), Av. Duque de Caxias Norte 225, Pirassununga, SP 13635-900, Brasil. Dissertação de Mestrado com apoio da CAPES. *Autor para correspondência: crisgtitto@usp.br

${ }^{3}$ Instituto de Ciências Agrárias e Ambientais Mediterrânicas, Universidade de Évora, Polo da Mitra, Apartado 94, Évora, 7002-554, Portugal.
}

lar se mostra como um indicador confiável para a condição de estresse pontual de suínos. 0 objetivo deste estudo foi determinar a relação entre medidas de termografia infravermelha da superfície ocular e de cortisol em situações de estresse em leitões na pós desmama. Foram avaliados 66 leitões, uma vez por semana, durante sete semanas, em dois períodos do dia ( $7 \mathrm{~h}$ e $15 \mathrm{~h}$ ) com medidas de temperatura superficial ocular, temperatura superficial do dorso e amostras de saliva para determinação de cortisol salivar. A análise estatística contemplou os efeitos fixos de semana e período do dia e sua interação e correlações de Pearson 
para relação entre termografia ocular, temperatura superficial e cortisol a 5\% de significância. Cortisol salivar não diferiu entre os períodos, mas foi superior nas três primeiras semanas após o desmame $(\mathrm{P}<0,05)$. Nas duas primeiras semanas após a desmama o cortisol apresentou correlação alta e positiva $(\mathrm{P}<0,05)$ com a temperatura ocular máxima $(0,89)$ e a temperatura superficial do dorso $(0,80)$. As duas temperaturas superficiais apresentaram uma associação moderada positiva $(\mathrm{r}=0,41 ; \mathrm{P}<0,0001)$ durante todo o período experimental. Este estudo destaca que a temperatura de superfície ocular obtida por meio da termografia infravermelha pode ser um indicador de temperatura de superfície corporal e estado de bem-estar de leitões em fase de creche, além de ser um método não invasivo e de rápida mensuração. Entretanto, mais pesquisas são necessárias para aprofundar a relação entre temperatura ocular e cortisol durante estresse crônico.

TERMOS DE INDEXAÇÃO: Termografia infravermelha, superfície ocular, indicador de estresse, suínos na fase de creche, bem-estar animal, cortisol salivar, desmama, imagem infravermelha, temperatura de superfície corporal.

\section{INTRODUÇÃo}

O uso de novas tecnologias na produção animal tem sido de grande importância para promover conforto e bem-estar nos animais. A termografia infravermelha é uma técnica não invasiva que permite mensurar a temperatura de superfície corporal sem conter o animal (Warriss et al. 2006).

O sistema nervoso central é o principal regulador de temperatura do corpo, e a temperatura do cérebro é reconhecida como a temperatura núcleo do sistema (McCafferty 2007). Estudos recentes com termografia infravermelha mostraram que a temperatura do olho pode ser um bom indicador da temperatura do núcleo, por sua proximidade como o cérebro, especificamente a temperatura de pequenas áreas em torno da margem da pálpebra e a carúncula lacrimal, que possuem uma relação estreita com a atividade simpática pelos leitos capilares inervados e respondem às mudanças no fluxo sanguíneo (Kessel et al. 2010; Johnson et al. 2011).

Em situações de estresse pontual, como no momento do pré-abate, já foi confirmada uma relação confiável entre a temperatura de superfície ocular medida pela termografia infravermelha e a concentração de lactato sanguíneo em leitões (Weschenfelder et al. 2013).

Já a fase pós-desmama é o período mais estressante para o leitão, caracterizado por um estresse prolongado. Em ambiente natural, o processo de desmame é feito de forma progressiva, com o leitão trocando lentamente seu alimento liquido pelo sólido (Newberry \& Wood-Gush 1985). Em sistemas comerciais, o desmame é feito de forma severa, com o reagrupamento de leitegadas e introdução abrupta de alimentação sólida (Pajor et al. 1991), além da separação materna. Neste processo é comum se observar aumento drástico das concentrações de cortisol no organismo e aumento de temperatura interna (Parrott \& Lloyd 1995).

No entanto, a relação entre estresse da desmama, concentração de cortisol salivar e temperatura de superfície ocular em suínos ainda não foi bem estabelecida. Desta for- ma, o objetivo deste estudo é determinar a relação entre medidas de termografia infravermelha na superfície ocular e cortisol salivar em leitões na fase de creche pós desmama.

\section{MATERIAL E MÉTODOS}

Foram utilizados 66 leitões machos e fêmeas durante a fase de creche, provindos de seis leitegadas nascidas dentro do mesmo mês (abril/2015) na suinocultura da Prefeitura do Campus Fernando Costa da Universidade de São Paulo, Pirassununga, SP. 0 projeto foi aprovado pela Comissão de Ética no Uso de Animais da Faculdade de Zootecnia e Engenharia de Alimentos (CEUA/FZEA no.5876090215). As baias experimentais possuem piso de cimento, 6 metros de comprimento por 2,5 metros de largura, laterais com uma altura de 1 metro, fechadas para limitar o contato físico e visual entre os leitões. 0 telhado possui telhas de cerâmica e o pé direito das instalações é de 3 metros, com ventilação natural e controle da mesma pela abertura e fechamento de cortinas, além de duas lâmpadas de $100 \mathrm{~W}$ ligadas $24 \mathrm{~h}$ para aquecimento dos animais.

Os leitões foram desmamados abruptamente aos 28 dias de idade no período da manhã, sendo pesados individualmente, vacinados contra paratifo dos leitões, pasteurelose, colibacilose, erisipela, rinite atrófica e leptospirose suína (Suiven ${ }^{\circledR}$ ) e receberam vermífugo. Neste mesmo dia foi iniciada a fase experimental. Os animais com peso médio de 7,28 $\pm 1,1 \mathrm{~kg}$ foram separados em grupos homogêneos com relação ao peso, sem controle em relação ao sexo e transferidos para as baias da creche, permanecendo até o 77으 dia de vida (a fase da creche teve uma duração de 49 dias). Os animais foram mantidos em seis baias com 11 animais cada uma, com acesso à agua e comida ad libitum. Foi realizado controle sanitário, sendo observadas ocorrências de diarreias entre a 1aㅡ semana até a 4⿳亠丷a semana de experimento e episódios de sintomatologia de pneumonia, observados apenas na 7ạ semana de experimento.

Foram realizadas sete colheitas de dados, com intervalos de sete dias em dois horários distintos (7:00 horas e 15:00 horas) para verificar um possível efeito do ritmo diurno do cortisol e da temperatura de superfície corporal, iniciando com a primeira colheita no dia da desmama. As colheitas de dados ocorreram sempre nas baias onde os animais estavam alojados, sem necessidade de contenção manual ou mudança de local.

A colheita de saliva para determinação do cortisol salivar foi realizada coletivamente por baia, deixando todos os animais da baia ou pelos menos $80 \%$ deles mastigar uma corda de algodão trançada durante 5 a 10 minutos. Após a obtenção da saliva necessária (1ml), a corda de algodão era prensada e as amostras de saliva eram armazenadas no Salivette ${ }^{\circledR}$, centrifugadas a 3500rpm por 30 minutos para uma limpeza das mesmas, e logo após os sobrenadantes eram enviados para análise em laboratório comercial para determinação do cortisol pelo método Eletroquímioluminescência (Escribano et al. 2012).

Para análise da temperatura de superfície corporal foram escolhidos aleatoriamente quatro animais por baia em cada colheita e registradas duas medidas por animal: Temperatura Superficial Dorsal (TSD) com termômetro infravermelho (Raynger Raytek ST ProPlus, USA) e Temperatura Superficial Ocular (TSO) utilizando uma câmera termográfica (Thermal imager TESTO $875^{\circledR}$, Lenzkirch, Alemanha) com coeficiente de emissividade de 0,98. A distância utilizada entre a câmera ou o termômetro infravermelho para o registro dos dados foi entre 0,50 e $1 \mathrm{~m}$. A luz infravermelha do termômetro era direcionada na parte dorsal do animal, percorrendo a área por três vezes, obtendo-se assim a temperatura média superficial instantaneamente. Dependendo da posição do animal foram registradas imagens do olho direito ou esquerdo. As ima- 

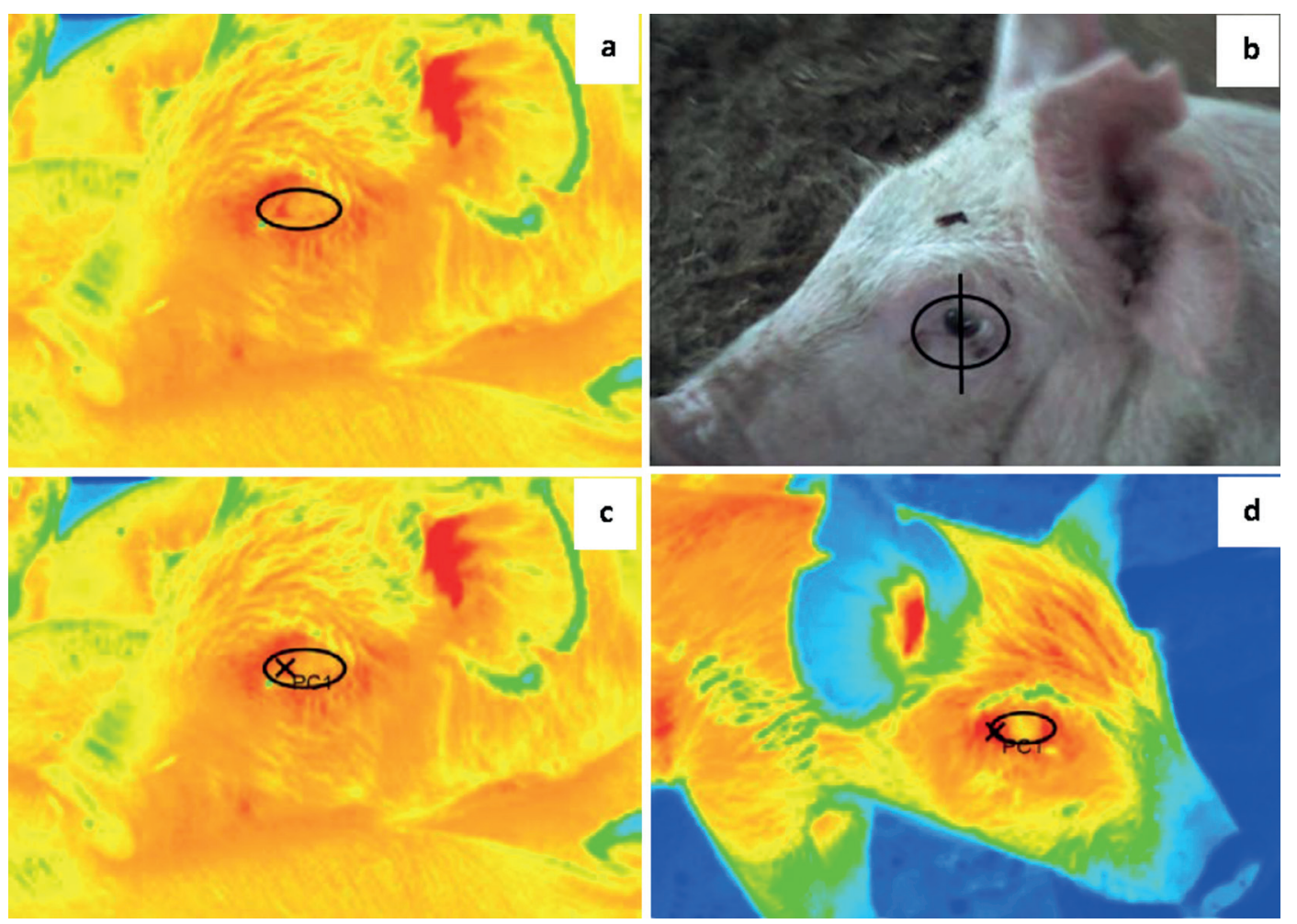

Fig.1. (a) Imagem termográfica do olho, (b) área determinada para análise, (c) ponto mais quente do olho (x) na posição interna próximo à carúncula lacrimal, e (d) na posição externa mais próxima à parte posterior do olho.

gens termográficas foram analisadas utilizando o software IRSoft Version 3.6 Testo Thermal Imagers para determinar temperatura máxima da área determinada entre a borda superior, média e inferior e a carúncula lacrimal (Fig.1a). Posteriormente, a partir da imagem de termografia infravermelha foi avaliada a localização da temperatura máxima, criando-se uma divisão vertical na área ocular previamente determinada na área do olho (Fig.1b), analisando duas posições: interna, da metade do olho até a carúncula lagrimal (Figura 1c); e externa, da metade do olho até a parte posterior do olho (Fig.1d) (Stewart et al. 2008, Johnson et al. 2011).

As variáveis meteorológicas: temperatura do ar, umidade relativa e a temperatura do globo negro foram registradas nas baias experimentais por meio de data logger (Onset $\mathrm{HOBO}^{\circledR} \mathrm{TEMP} /$ $\mathrm{RH} / 2$ ext channels) a cada 15 minutos, instalados no centro das baias a uma altura de 1,5 $\mathrm{m}$ do chão. 0 dispositivo permaneceu no local durante todo o período experimental (Quadro 1).

Os dados de TSO e TSD foram analisados com efeitos fixos de semana (1-7) e período (7 e 15 horas) e suas interações. As comparações de médias foram realizadas pelo teste $\mathrm{F}$ e teste $\mathrm{t}$ (PDIFF) utilizando o procedimento GLM do software SAS 9.2 (SAS Inst. Inc., Cary., NC). Para se relacionar os dados de temperatura superficial ocular por termografia infravermelha, temperatura de superfície dorsal e o cortisol salivar utilizou-se o coeficiente de correlação de Pearson dentro de cada semana e no período total (CORR, SAS). Foi determinada a posição ocular (interna ou externa) da temperatura máxima pelo teste-t. 0 nível de probabilidade de $P<0,05$ foi escolhido como limite para significância estatística em todos os testes.

\section{RESULTADOS E DISCUSSÃO}

Para os dados de TSO máxima houve diferença entre os períodos na primeira $(\mathrm{P}<0,01)$, segunda $(\mathrm{P}<0,01)$, e sexta $(\mathrm{P}=0,045$; Quadro 2) semana de creche, encontrando-se no período da manhã valores menores em relação ao período da tarde. Dentro do período da manhã, as duas primeiras semanas apresentaram as temperaturas mais baixas, sendo diferentes entre si e entre as semanas seguintes $(\mathrm{P}<0,05)$. Já da $33^{\underline{a}}$ até a $7 \underline{\text { a }}$ semana as médias foram semelhantes $(\mathrm{P}>0,05)$. Entretanto, para o período da tarde, a 1aㅡ $3^{\underline{a}}$ e $7^{\mathrm{a}}$ semanas apresentaram valores médios de temperatura menores, indicando diferença com a $5^{\underline{a}}$ e $6^{\underline{a}}$ semana $(\mathrm{P}<0,05)$.

Quadro 1. Valores médios, máximos e mínimos das variáveis meteorológicas durante o período experimental

\begin{tabular}{|c|c|c|c|c|c|c|c|c|}
\hline \multirow[b]{2}{*}{ Mês } & \multicolumn{2}{|c|}{$\begin{array}{c}\text { Temperatura } \\
\text { do ar }\left({ }^{\circ} \mathrm{C}\right)\end{array}$} & \multicolumn{3}{|c|}{$\begin{array}{c}\text { Temperatura do } \\
\text { globo negro }\end{array}$} & \multicolumn{3}{|c|}{$\begin{array}{l}\text { Umidade } \\
\text { relativa (\%) }\end{array}$} \\
\hline & Média Máx. & Mín. & Média & Máx. & Mín. & Média & Máx. & Mín. \\
\hline bri & 24,25 & 14,98 & 24,15 & 34,87 & 16,05 & 75,05 & 92,98 & 35,48 \\
\hline Maio & 22,17 & 14,38 & 22,64 & 33,28 & 16,10 & 76,74 & 93,51 & 37,28 \\
\hline Junho & 21,32 & 13,58 & 21,79 & 33,4 & 15,3 & 77 & 94,2 & 34 \\
\hline
\end{tabular}

Quadro 2. Médias seguidas pelo erro padrão médio da temperatura ocular máxima em leitões para cada semana na fase de creche por período do dia

\begin{tabular}{cccc}
\hline Semana & Manhã & Tarde & Valor P entre períodos \\
\hline 1 & $36,09(0,094)^{\mathrm{c}}$ & $37,65(0,093)^{\mathrm{b}}$ & $<0,001$ \\
2 & $36,64(0,194)^{\mathrm{b}}$ & $37,78(0,203)^{\mathrm{ab}}$ & $<0,001$ \\
3 & $37,48(0,190)^{\mathrm{a}}$ & $37,63(0,203)^{\mathrm{b}}$ & NS \\
4 & $37,43(0,194)^{\mathrm{a}}$ & $37,91(0,190)^{\mathrm{ab}}$ & NS \\
5 & $37,80(0,194)^{\mathrm{a}}$ & $38,13(0,190)^{\mathrm{a}}$ & NS \\
6 & $37,62(0,194)^{\mathrm{a}}$ & $38,17(0,190)^{\mathrm{a}}$ & 0,045 \\
7 & $37,47(0,190)^{\mathrm{a}}$ & $37,61(0,186) \mathrm{b}$ & NS
\end{tabular}

Letras minúsculas difęrentes na coluna diferem entre si pelo Teste-t (PDIFF) a $5 \%$. NS = não significativo 


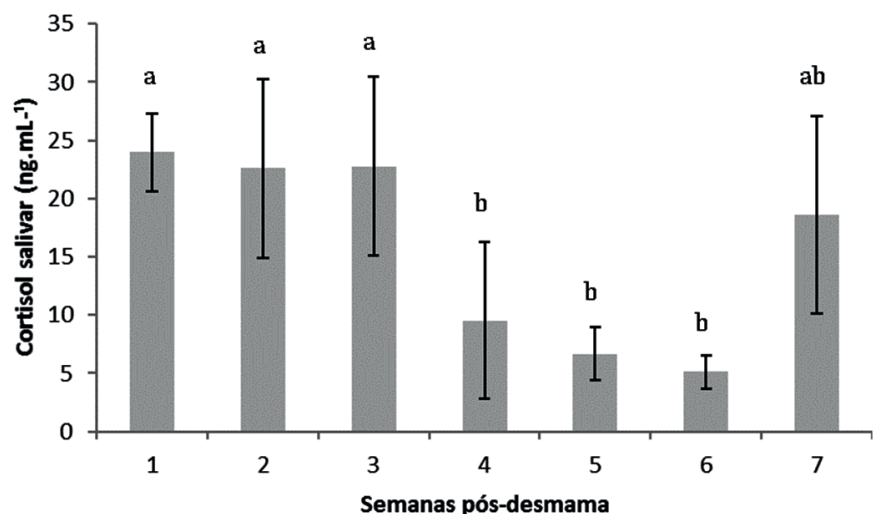

Fig.2. Médias e erro padrão médio da concentração de cortisol salivar em leitões para cada semana pós-desmama na fase de creche.

As demais semanas foram semelhantes a todas $(\mathrm{P}>0,05)$. A vasoconstrição pode ser uma resposta do animal ao estresse, aumentando assim a temperatura corporal profunda e diminuindo a periférica, denominado como hipertermia induzida pelo estresse (Cabanac \& Briese 1992), produzindo temperaturas baixas no olho. É provável que, nas semanas com menor temperatura de superfície ocular máxima, os animais se encontravam sofrendo algum tipo de estresse seja por motivo da desmama, ou por causa da pneumonia presente na 7a semana de creche. Diversos estudos demostram que bovinos (Stewart et al. 2007) e ovinos (Stubsjøen et al. 2009) em uma situação de estresse pode diminuir a temperatura do olho.

Os dados de cortisol salivar não apresentaram efeito de período $(\mathrm{P}>0,05)$, com valores semelhantes para manhã e tarde. Sabe-se que em suínos, que são animais diurnos, as concentrações de glicocorticóides são mais elevadas pela manhã e diminuídos durante a noite (Janssens et al. 1995, Hillmann et al. 2008), entretanto, durante um período de estresse crônico a variação circadiana do cortisol pode diminuir (Janssens et al. 1995, De Jong et al. 2000). Na semana da desmama foi observada a média mais alta de cortisol salivar, concentrações mantidas elevadas na segunda e terceira semanas ( $P>0,05$, Fig.2), diferente das semanas subsequentes, onde as concentrações diminuíram $(\mathrm{P}<0,05)$. Na última semana da creche foi notado um novo aumento de cortisol salivar, porém semelhante com todas as semanas $(\mathrm{P}>0,05)$. A desmama é um dos manejos mais severos da produção de suínos e é uma das atividades que mais causa estresse nos leitões (Fraser et al. 1991). Os resultados mostram claramente um aumento no cortisol salivar após o desmame, frente a valores basais para a espécie entre 5,1 e 6,8ng. $\mathrm{mL}^{-1}$ (Dalla costa et al. 2008, Pulido-Rodriguez 2016). Mudanças do ambiente, reagrupamento de lotes e manuseio continuo e prologando dos animais aumenta o estresse dos animais comprometendo seu sistema imunológico (Manteuffel 2002, Tuchscherer et al. 2002). Todavia, a partir da $4^{\underline{a}}$ semana, quando episódios de diarreia já costumam ser menos frequentes, os valores de cortisol salivar diminuíram, voltando a se elevar na 7 a semana, quando foram observados episódios de pneumonia nos grupos.

As correlações entre as TSD e TSO máxima para suínos na fase de creche foram moderadas $(0,41)$, indicando que o aumento da TSD está associado com o aumento da TSO $(\mathrm{P}<0,05)$. A TSD pode ser uma boa medida para descrever o ambiente no qual o animal está inserido (Weschenfelder et al. 2013, Stewart et al. 2008, Collier et al. 2006).

Houve correlação alta e positiva $(0,89)$ entre o cortisol salivar e a TSO máxima, respectivamente para a primeira e segunda semana pós-desmama $(\mathrm{P}<0,05)$, assim como para a TSD $(0,80)$ e cortisol. Por outro lado, a partir da terceira semana não foram observadas correlações significativas $(\mathrm{P}>0,05)$ entre o cortisol e a TSO máxima e TSD. Quando se observa o perfil de variação da temperatura ocular máxima ao longo das semanas é possível notar uma relação nas primeiras semanas, quando o cortisol é mais elevado devido à separação da mãe. A alteração da temperatura ocular pode ser mediada por um componente simpático do sistema nervoso autônomo, atividade relacionada a uma das primeiras fases da resposta ao estresse (Stewart et al. 2010). Na literatura há divergências sobre a relação do aumento do cortisol com a temperatura ocular. A maior parte dos estudos é relacionada com o estresse pontual, que apresenta correlações positivas entre cortisol e temperatura ocular, todavia a mudança de fase em leitões (maternidade para creche) pode ser considerada um estresse crônico e desta forma não seguir este padrão de correlação ao longo do tempo. Quando um animal se torna estressado, o eixo hipotálamo-pituitária-adrenal é ativado e calor é produzido como resultado do aumento da concentração de cortisol, realizando mudanças na produção e perda de calor no animal (Schaefer et al. 2002). Em suínos houve aumento de cortisol após manejo diário e castração, porém, só foi observado aumento de temperatura ocular após castração, com correlação positiva (Lonardi et al. 2015). Foi observado aumento em ambos os parâmetros após cateterismo em vacas (Stewart et al. 2007) e em bezerros recém descornados foi observada diminuição da temperatura ocular após 5 minutos da aplicação de ACTH e posteriormente houve um aumento, vinculado ao pico de cortisol (Stewart et al. 2008). Em cavalos, durante corte de crina, houve correlação positiva entre cortisol e temperatura máxima do olho entre 10 e 20 minutos após o procedimento (Yarnell et al. 2013) e durante competições (Valera et al. 2012).

Encontrou-se TSO máxima mais próxima da carúncula lacrimal com média de $37,41^{\circ} \mathrm{C}$ e menores temperaturas na parte externa do olho $\left(37,13^{\circ} \mathrm{C} ; \mathrm{P}<0,05\right)$. Quando ocorre a ativação do eixo hipotálamo-hipófise-adrenal os níveis de cortisol aumentam como alterações do fluxo sanguíneo, o que promove as mudanças na produção de calor, o que pode ser avaliado pela termografia ocular (Church et al. 2009). A temperatura ocular pode estar associada à temperatura do núcleo corporal por sua proximidade ao cérebro. Por razões anatômicas, o olho e a parte de atrás das orelhas são pontos confiáveis para medição de temperatura de superfície corporal por meio da termografia (Schmidt et al. 2013). Alguns estudos em bovinos (Stewart et al. 2008) e equinos (Johnson et al. 2011, Dai et al. 2015) também observaram a posição da TSO máxima perto da carúncula lacrimal.

Para os suínos, a mensuração de variáveis fisiológicas como a temperatura retal se torna delicada por necessitar 
conter os animais, e a dosagem de cortisol salivar não é uma medida instantânea da condição fisiológica do animal, portanto, a termografia infravermelha é uma boa alternativa não invasiva e de rápida análise. Já é sabido que a temperatura de superfície corporal na região do dorso possui alta correlação $(0,90)$ com a temperatura retal (Mostaco 2014), desta forma, valores de temperatura infravermelha ocular podem ser um indicador de temperatura corporal e um método útil para avaliar a condição fisiológica de estresse em suínos (Weschenfelder et al. 2013), sendo um bom substituto para outras metodologias invasivas (Johnson et al. 2011, Brown-Brandl et al. 2013). A indicação de correlações moderadas entre TSO, TSD e cortisol aponta para uma possível relação entre temperatura de superfície corporal e o estresse causado pelo manejo da desmama (Beausoleil et al. 2004, Drew 1996, MacAulay et al. 1995, Parrott et al. 1988). Desta forma, o uso da termografia infravermelha pode ser uma ferramenta não invasiva que fornece medidas práticas que podem estar associadas também com outros parâmetros fisiológicos e hormonais, como frequência respiratória e cortisol, para avaliar o bem-estar de leitões (Weschenfelder et al. 2013, Stewart et al. 2010, 2008, Collier et al. 2006).

\section{CONCLUSÕES}

Este estudo destaca que a temperatura de superfície ocular obtida por meio da termografia infravermelha pode ser um indicador de temperatura corporal e estado de bem- estar de leitões em fase de creche, além de ser um método não invasivo e de rápida mensuração.

Entretanto, mais pesquisas são necessárias para aprofundar a relação entre temperatura ocular e cortisol durante estresse crônico.

Agradecimentos.- Agradecemos à Dra. Thays Mayra da Cunha Leme-dos-Santos pelo apoio no desenvolvimento do estudo.

\section{REFERÊNCIAS}

Beausoleil N.J., Stafford K.J. \& Mellor D.J. 2004. Can we use change in core body temperature to evaluate stress in sheep? Proc. N.Z. Soc. Anim. 64:72-76.

Brown-Brand T., Eigenber R. \& Purswell J. 2013. Using thermal imaging as a method of investigating thermal thresholds in finishing pigs. Biosyst. Eng. 114: 327-333.

Cabanac A. \& Briese E. 1992. Handling elevates the colonic temperature of mice. Physiol Behav. 51:95-98.

Collier R.J., Dahl G.E. \& Vanbaale M.J. 2006. Major advances associated with environmental effects on dairy cattle. J. Dairy Sci. 89:1244-1253.

Church J.S., Cook N.J. \& Schaefer A.L. 2009. Recent applications of infrared thermography for animal welfare and veterinary research: everything from chicks to elephants. InfraMation. Proceedings... Disponível em $<$ http://www1.infraredtraining.com/view/?id=40284> Acesso em 10 dez. 2015

Dai F., Cogi N.H., Heinzl E.U.L., Dalla Costa E., Canali E. \& Minero M. 2015. Validation of a fear test in sport horses using infrared thermography. J. Vet. Behav. 10:128-136

Dalla Costa O.A., Ludke J.V., Paranhos da Costa M.J.R., Faucitano L., Coldebella A., Kich J.D., Peloso J.V. \& Roza D.D. 2008. Tempo de jejum na granja sobre o perfil hormonal e os parâmetros fisiológicos em suínos de abate pesados. Ciência Rural 38(8):2300-2306.

De Jong I.C., Prelle I.T., van de Burgwal J.A., Lambooij E., Korte S.M., Blokhuis H.J. \& Koolhaas J.M. 2000. Effects of environmental enrichment on behavioral responses to novelty, learning, and memory, and the circadian rhythm in cortisol in growing pigs. Physiol. Behav. 68(4):571-578.

Drew M.L. 1996. The use of a tympanic membrane thermometer for assessing hyperthermia in bighorn sheep. J. Wildl. Dis. 32:512-516.

Escribano D., Fuentes-Rubiano M. \& Cerón J.J. 2012. Validation of an automated chemiluminescent immunoassay for salivary cortisol measurements in pigs. J. Vet. Diagn. Invest. 24:918-923.

Fraser D., Phillips P.A., Thompson B.K. \& Tennessen T. 1991. Effects of straw on the behaviour of growing pigs. Appl. Anim. Behav. Sci. 30:307-318.

Hillmann E., Schrader L., Mayer C. \& Gigax L. 2008. Effects of weight, temperature and behaviour on the circadian rhythm of salivary cortisol in growing pigs. Anim. Consortium 8:405-409.

Janssens C.J., Helmond F.A. \& Wiegant V.M. 1995. The effect of chronic stress on plasma cortisol concentrations in cyclic female pigs depends on the time of day. Domest. Anim. Endocrinol. 12:167-177.

Johnson S.R., Rao S., Hussey S.B., Morley P.S. \& Traub-Dargatz J.L. 2011. Thermographic eye temperature as an index to body temperature in ponies. J. Equine Vet. Sci. 31:63-66.

Kessel L., Johnson L., Arvidsson H. \& Larsen M. 2010. The relationship between body and ambient temperature and corneal temperature. Invest. Ophthamol. Vis. Sci. 51:6593-6597.

Lonardi C., Scollo A., Normando S., Brscic M. \& Gottardo F. 2015. Can novel methods be useful for pain assessment of castrated piglets? Animal 9 (5):871-877.

Macaulay A.S., Hahn G.L., Clark D.H. \& Sisson D.V. 1995: Comparison of calf housing types and tympanic temperature rhythms in Holstein calves. J. Dairy Sci. 78:856-862.

Manteuffel G. 2002. Central nervous regulation of the hypothalamic-pituitary-adrenal axis and its impact on fertility, immunity, metabolism and animal welfare - a review. Archiv für Tierzucht 45(6):575-595.

McCafferty D.J. 2007. The value of infrared thermography for research on mammals: previous applications and future directions. Mamm. Rev. 37:207-223.

Mostaco G.M. 2014. Determinação da temperatura retal e frequência respiratória de suínos em fase de creche por meio da temperatura da superfície corporal em câmara climática. Dissertação de Mestrado. Disponível em <http.tesesonline.usp.br> Acesso em 12 abr. 2016.

Newberry R.C. \& Wood-Gush D.G.M. 1985. The suckling behaviour of domestic pigs in a semi-natural environment. Behav. 95:11-25.

Pajor E.A., Fraser D. \& Kramer D.L. 1991. Consumption of solid food by suckling pigs: individual variation and relation to weight gain. Appl. Anim. Behav. Sci. 32:139-155.

Parrott R.F., Bradshaw R.H., Lloyd D.M. \& Goode J.A. 1988. Effects of transport and indomethacin on telemetered body temperature and release of cortisol and prolactin in pre-pubertal pigs. Res. Vet. Sci. 64:51-55.

Parrott R.F. \& Lloyd D.M. 1995. Restraint, but not frustration, induces prostaglandin mediated hyperthermia in pigs. Physiol. Behav. 57:1051-1055.

Pulido-Rodriguez L.F. 2016. Bagaço de cana como enriquecimento ambiental para leitões recém desmamados. Dissertação de Mestrado. Disponível em <http.tesesonline.usp.br> Acesso em 12 abr. 2016.

Schaefer A.L., Matthews L.R., Cook N.J., Webster J. \& Scott S.L. 2002. Novel non-invasive measures of animal welfare. In: Animal Welfare and Behaviour. Science Solution, Joint NAWAC/ISAE Conference, 27-28 Jun. 2002, Hamilton, New Zealand.

Schmidt M., Hoffmann G., Ammon C., Schön P., Manteuffel C. \& Amon T. 2013. Application of infrared thermography on lactating sows. Livest. Machinery 68(4):228-231.

Stubsjøen S., Flø A., Moe R., Janczak A., Skjerve E., Valle P. \& Zanella A. 2009. Exploring non-invasive methods to assess pain in sheep. Physiol. Behav. 98:640-648.

Stewart M., Webster J.R., Verkerk G.A., Schaefer A.L., Colyn J.J. \& Stafford K.J. 2007. Non-invasive measurement of stress in dairy cows using infrared thermography. Physiol. Behav. 92(3):520-525.

Stewart M., Stafford K.J., Dowling S.K., Schaefer A.L. \& Webster J.R. 2008. Eye temperature and heart rate variability of calves disbudded with or without local anaesthetic. Physiol. Behav. 93(4/5):789-797. 
Stewart M., Webster J.R., Stafford K.J., Schaefer A.L. \& Verkerk G.A. 2010. Effects of an epinephrine infusion on eye temperature and heart rate variability in bull calves: technical note. J. Dairy Sci. 93(11):52525257.

Tuchscherer M., Kanitz E., Otten W. \& Tuchscherer A. 2002. Effects of prenatal stress on cellular and humoral immune responses in neonatal pigs. Vet. Immunol. Immunopathol. 86(3/4):195-203.

Valera M., Bartolomé E., Sánchez M.J., Molina A., Cookc N. \& Schaefe A. 2012. Changes in eye temperature and stress assessment in horses during show jumping competitions. J. Equine Vet. Sci. 32:827-830.
Warriss P.D., Pope S.J., Brown S.N., Wilkings L.J. \& Knowles T.G. 2006. Estimating the body temperature of groups of pigs by thermal imaging. Vet. Rec. 158:331-334.

Weschenfelder A.V., Saucier L., Maldague X., Rocha L.M., Shaefer A.L. \& Faucitano L. 2013. Use of infrared ocular thermography to assess physiological conditions of pigs prior to slaughter and predict pork quality variation. Meat Sci. 95(3):616-620.

Yarnell K., Hall C. \& Billett E. 2013. An assessment of the aversive nature of an animal management procedure (clipping) using behavioral and physiological measures. Physiol. Behav. 118:32-39. 